\title{
Ionic skin
}

\section{Citation}

Sun, Jeong-Yun, Christoph Keplinger, George M. Whitesides, and Zhigang Suo. 2014. “Ionic Skin." Advanced Materials 26 (45) (October 29): 7608-7614. doi:10.1002/adma.201403441.

\section{Published Version}

doi:10.1002/adma.201403441

\section{Permanent link}

http://nrs.harvard.edu/urn-3:HUL.InstRepos:13943567

\section{Terms of Use}

This article was downloaded from Harvard University's DASH repository, and is made available under the terms and conditions applicable to Open Access Policy Articles, as set forth at http:// nrs.harvard.edu/urn-3:HUL.InstRepos:dash.current.terms-of-use\#OAP

\section{Share Your Story}

The Harvard community has made this article openly available.

Please share how this access benefits you. Submit a story.

\section{Accessibility}




\section{WILEY-VCH}

DOI: $10.1002 /(($ please add manuscript number $))$

Article type: Communication

\section{Ionic skin}

Jeong-Yun Sun, Christoph Keplinger, George M. Whitesides*, and Zhigang Suo*

Prof. J.-Y. Sun, ${ }^{[+]}$

1 Kavli Institute for Bionano Science and Technology, Harvard University, Cambridge MA 02138,USA.

2 School of Engineering and Applied Sciences, Harvard University, Cambridge, MA 02138, USA.

3 Department of Materials Science and Engineering, Seoul National University, Seoul, 151-744

Korea.

Dr. C. Keplinger, ${ }^{[+]}$

1 Kavli Institute for Bionano Science and Technology, Harvard University, Cambridge MA 02138,USA.

2 School of Engineering and Applied Sciences, Harvard University, Cambridge, MA 02138, USA.

3 Department of Chemistry and Chemical Biology, Harvard University, Cambridge, MA 02138, USA.

Prof. G.M. Whitesides

1 Kavli Institute for Bionano Science and Technology, Harvard University, Cambridge MA 02138,USA.

2 Department of Chemistry and Chemical Biology, Harvard University, Cambridge, MA 02138, USA.

3 Wyss Institute for Biologically Inspired Engineering, Harvard University, Cambridge MA 02138, USA.

E-mail: gwhitesides@gmwgroup.harvard.edu

Prof. Z. Suo

1 Kavli Institute for Bionano Science and Technology, Harvard University, Cambridge MA 02138,USA.

2 School of Engineering and Applied Sciences, Harvard University, Cambridge, MA 02138, USA. Email:suo@seas.harvard.edu

${ }^{[+]}$The first two authors contributed equally to this work.

Keywords: Strain sensors, pressure sensors, stretchable electronics, ionic conductors, electronic skin. 


\section{WILEY-VCH}

Our skin is a stretchable, large-area sheet of distributed sensors. These properties of skin have inspired the development of mimics, with differing levels of sophistication, to enable wearable or implantable electronics for entertainment and healthcare. ${ }^{[1-4]}$ "Electronic skin" is generally taken to be a stretchable sheet with area above $10 \mathrm{~cm}^{2}$ carrying sensors for various stimuli, including deformation, pressure, light and temperature. The sensors report signals through stretchable electrical conductors ${ }^{[5]}$ (e.g., carbon grease, ${ }^{[6]}$ microcraked metal films, ${ }^{[1]}$ serpentine metal lines, ${ }^{[2]}$ graphene sheets, ${ }^{[7]}$ carbon nanotubes, ${ }^{[8-10]}$ silver nanowires, ${ }^{[11]}$ gold nanomeshes, ${ }^{[12]}$ and liquid metals $\left.{ }^{[13,14]}\right)$. These conductors transmit signals using electrons. They meet the essential requirements of conductivity and stretchability, but struggle to meet additional requirements in specific applications, such as biocompatibility in biometric sensors, ${ }^{[15]}$ and transparency in tunable optics. ${ }^{[16,17]}$

By contrast, sensors in our skin report signals using ions. Here we explore the potential of ionic conductors in the development of a new type of sensory sheet, which we call "ionic skin". The sensory sheet is highly stretchable, transparent, and biocompatible. It readily monitors large deformation, such as that generated by the bending of a finger. It detects stimuli with wide dynamic range (strains from $1 \%$ to $500 \%$ ). It measures pressure as low as $1 \mathrm{kPa}$, with small drift over many cycles. A sheet of distributed sensors covering a large area can report the location and pressure of touch. High transparency allows the sensory sheet to transmit electrical signals without impeding optical signals.

Many ionic conductors, such as hydrogels and ionogels, are highly stretchable and transparent. ${ }^{[18-20]}$ These gels are polymeric networks swollen with water or ionic liquids. They behave like elastic solids and eliminate the need for containers as required in the case of liquid metal conductors. Whereas familiar elastic gels, such as Jell-O, are brittle and easily rupture, the recent decade has seen the development of hydrogels and ionogels as tough as elastomers. ${ }^{[20-}$ 22] Many hydrogels are biocompatible. They can be made softer than tissues, achieving the "mechanical invisibility" required for biometric sensors, which monitor soft tissues without 


\section{WILEY-VCH}

constraining them. Although most hydrogels dry out in open air, hydrogels containing humectants retain water in environment of low humidity, and ionogels are nonvolatile in vacuum. ${ }^{[18-20]}$

We have recently used ionic conductors-together with stretchable and transparent dielectrics-to make actuators, which deform in response to high voltages, on the order of kilovolts. ${ }^{[18]}$ By contrast, the sensors described here deform in response to applied forces, giving signals that can be measured using voltages below 1 volt. To illustrate principles in our design of the ionic skin, consider a simple example-a dielectric sandwiched between two ionic conductors (Figure 1). In many applications, the ionic skin reports signals ultimately to external electronic equipment. We form a hybrid ionic-electronic circuit by connecting the ionic skin to electronic conductors, in regions outside the active area of the sensory skin, using thin lines of the ionic conductors. These ionic interconnects mimic the function of axons, and can be as long as meters, if required by an application. ${ }^{[18]}$ Both the dielectric and the ionic conductors are stretchable and transparent, whereas the electronic conductors can be made of stiff and opaque metals. This design allows a large-area sheet of distributed sensors to be highly stretchable and transparent.

To allow repeated use over a long time, we design the hybrid ionic-electronic circuit to transmit electrical signals without electrochemical reaction. Electrical double layers form at the interfaces between the electrodes and the ionic conductors, as well as at the interfaces between the ionic conductors and the dielectric. ${ }^{[23]}$ For many combinations of electrodes and ionic conductors, when no voltage is applied between the two electrodes, the layered structure reaches a state of thermodynamic equilibrium, the "voltage-off" state (Figure 1a). When a voltage is applied between the two electrodes, (so long as the additional voltage across the interface between the electrode and ionic conductor is within a range, e.g., between $-1 \mathrm{~V}$ and $+1 \mathrm{~V}$ ), electrons and ions do not cross the interface, no electrochemical reaction occurs, and the structure reaches a new state of thermodynamic equilibrium, the "voltage-on" state (Figure 1b). 


\section{WILEY-VCH}

The applied voltage causes additional electric charges and potential through the layered structure (Figure S1). The electrical double layer at each interface between a metallic electrode and an ionic conductor behaves like a capacitor, in series with the capacitor due to the dielectric (Figure 1c). The capacitance $C$ measured between the two electrodes relates to the capacitance of the dielectric $C_{\mathrm{D}}$ and the capacitances of the electrical double layers $C_{\mathrm{EDL}}$ as $1 / C=2 / C_{\mathrm{EDL}}+1 / C_{\mathrm{D}}$ Charges in the electrode and in the ionic conductor are separated over nanometers, but charges on the two faces of the dielectric are separated by its thickness (on the order of $0.1 \mathrm{~mm}$ in our experiments). Consequently, the electrical double layer has a large capacitance compared to the dielectric, $C_{\mathrm{EDL}} / C_{\mathrm{D}} \sim 10^{5}$, and the measured capacitance is dominated by that of the dielectric, $C \sim C_{\mathrm{D}}$. Nearly all the voltage applied between the two electrodes drops across the dielectric, and the additional voltage across the electrical double layer is much smaller than $1 \mathrm{~V}$. The fact that the voltage across the electrical double layer is small prevents electrochemical reaction.

When external forces deform the dielectric, the capacitance of this part of the circuit increases (Figure 1d, 1e). A measurement of this change in capacitance enables the ionic skin to sense the deformation. When a hydrogel serves as a resistive strain sensor, the use of lowvoltage AC signals to measure impedance avoids electrochemical reactions. ${ }^{[24]}$ By contrast, our design averts electrochemical reactions by taking advantage of the orders-of-magnitude higher capacitance of the electrical double layers relative to the dielectric. The ionic skin is, thus, a capacitive sensor. It is known that capacitive sensors achieve the highest precision of all electrical sensors, have simple and robust structures, feature high sensitivity and resolution, and allow long-term, drift-free sensing even when temperature changes. ${ }^{[25-28]}$

We next derive the relation between deformation and capacitance. We adopt the model of ideal dielectric elastomers, assuming that the volume and permittivity remain constant as the elastomers deform. ${ }^{[29]}$ When a dielectric sheet is stretched by factors $\lambda_{1}$ and $\lambda_{2}$ in its plane, the 


\section{WILEY-VCH}

thickness of the sheet scales by factor $\lambda_{3}=1 / \lambda_{1} \lambda_{2}$, and the capacitance $C$ of the dielectric scales as $C=C_{0}\left(\lambda_{1} \lambda_{2}\right)^{2}$, where $C_{0}$ is the capacitance of the dielectric in the undeformed state. When a uniaxial force stretches a dielectric to $\lambda$ times its initial length, both the width and the thickness of the dielectric reduce by a factor $\sqrt{\lambda}$, and the capacitance of the dielectric scales as $C=C_{0} \lambda$. When equibiaxial forces stretch a dielectric to $\lambda$ times its initial length in both directions, the capacitance of dielectric elastomer scales as $C=C_{0} \lambda^{4}$.

We demonstrated a strain sensor by using a polyacrylamide hydrogel containing $\mathrm{NaCl}$ as the ionic conductor, and an acrylic elastomer (VHB 4905, 3M) as the dielectric (Figure 2). Both materials are highly stretchable and transparent. VHB is marketed as a double-sided adhesive tape. The adhesion between the ionic skin and the finger was adequate and no debonding occurred. To limit the evaporation of water from the hydrogels, we covered them with two layers of VHB (Figure 2a).

The softness of the ionic skin allows it to conform readily to dynamic, curved surfaces. We attached the ionic skin on a straight finger (Figure $2 b$ ). When the finger bent, the ionic skin stretched and followed to the movement of the joint (Figure 2c). As the finger bent repeatedly, we recorded the capacitance of the ionic skin using a simple capacitance meter (LCR/ESR meter, Model 885, BK Precision), set to a sinusoidal measurement signal of $1 \mathrm{~V}$ and $100 \mathrm{~Hz}$ (Figure 2d, Supplementary video 1). During large deformations, the ionic skin remained adherent to the finger and highly transparent (Figure 2e).

The strain sensor has a large dynamic range and is stable over more than 1000 cycles. We clamped our device at each end between acrylic plates (Figure S2), stretched it using a mechanical testing apparatus (Instron, Model 3342) with a $50 \mathrm{~N}$ load cell at a strain rate of $0.5 / \mathrm{min}$, and measured the capacitance using a capacitance meter (Agilent, E4980A) with a voltage of $1 \mathrm{~V}$ at a frequency of $20 \mathrm{kHz}$. The ionic skin could be stretched to about six times the initial length of the strip (Figure 3a,b). The experimentally measured capacitance increased 


\section{WILEY-VCH}

linearly with the stretch, and matched well with the theoretical prediction, $C=C_{0} \lambda$ (Figure 3c).

At large stretches near rupture, the measured capacitance was slightly lower than that of the theoretical prediction. We did not observe any sliding and fracture, and attributed the deviation of the capacitance to a lowering of the permittivity of the dielectric at large strain.[3о] We also stretched the ionic skin by cyclic uniaxial force at a frequency of $1 \mathrm{~Hz}$ and a maximum strain of $2 \%$. The change in capacitance of the sensor followed the change in strain, and the drift of capacitance was within $5 \%$ over 4000 cycles (Figures 3d, S3).

We designed and fabricated a stretcher to pull an ionic skin under equibiaxial conditions (Figure 3e, 3f, S4, Supplementary video 2). When the stretcher fixed the ionic skin to a state of biaxial stretch, we took a photo and determined the level of stretch by analyzing the area of the dielectric covered by the conductors. We fabricated strain sensors using VHB and two types of conductors: hydrogel and carbon grease. These strain sensors exhibited nearly identical capacitance-stretch curves (Figure 3g). At high stretches, the measured capacitances of the strain sensors were somewhat lower than the theoretical prediction, $C=C_{0} \lambda^{4}$, which can again be attributed to a lowering of the permittivity of the dielectric at high strain.

The ionic skin also readily functions as a pressure sensor. We fabricated a pressure sensor of dimensions $10 \times 10 \times 2.4 \mathrm{~mm}^{3}$ (Figure 4a). The pressure sensor was placed between two grounded metal stages, and a small amount of mineral oil was applied on the surfaces of the sensor to reduce the adhesion and friction between the sensor and the stages (Figure 4b). As the mechanical testing apparatus compressed the sensor at a strain rate of $0.5 / \mathrm{min}$, the capacitance of the sensor was measured. The two layers of the hydrogel raised the height of the pressure sensor relative to the surrounding elastomer, so that the testing machine applied the force on the pressure sensor. As the pressure sensor was compressed in thickness, its area expanded. The expansion of the pressure sensor was somewhat constrained by the surrounding elastomer. This constraint stiffened the response of the pressure sensor, but still allowed significant change 


\section{WILEY-VCH}

in capacitance (Figure 4c). The pressure was defined as the applied force divided by the area of the undeformed dielectric elastomer. The pressure sensor was also compressed repeatedly with a prescribed maximum strain of $1 \%$ at a frequency $0.5 \mathrm{~Hz}$, while the capacitance and pressure were recorded (Figure 4d). The capacitance-pressure curves were stable over 1000 cycles (Figure 4e, S5). The small reduction in pressure over the cycles was possibly due to viscoelasticity of the materials.

We fabricated a sheet of distributed sensors to demonstrate the capability of detecting the location and pressure of touch. The demonstration consisted of four small squares of hydrogel, which lay over a sheet of dielectric, which in turn lay over a single large layer of grounded hydrogel (Figure 5a). A rotational switch connected the four sensors, one at a time, to a capacitance meter (Agilent, E4980A) (Figure 5b), which was controlled by a LabVIEW (National Instruments) program. We attached the sheet on the back of a hand, and pressed the sensors with a finger (Figure 5c). The ionic skin detected the location of touch (Figure $5 \mathrm{~d}$, Supplementary Video 3). The ionic skin readily resolved the pressure of a gentle touch of a finger $(<10 \mathrm{kPa})^{[31]}$ Whereas an on/off button expresses two states, a sensor in our sensory sheet continuously measures the level of pressure (Figure 5e, Supplementary Video 4).

We have used ionic conductors to demonstrate a new type of sensory sheets. They are highly stretchable, transparent, and biocompatible. This unique combination of attributes will open doors to applications in wearable or implantable electronics. In particular, high transparency will allow the sensory sheets to transmit electrical signals without impeding optical signals. This property will enable optical stimulation, as well as continuous inspection of the surfaces covered by the sensory sheets.

\section{Experimental Section}

The hydrogels were synthesized using acrylamide (AAm; Sigma, A8887) as monomers, N,N-methylenebisacrylamide (MBAA; Sigma, M7279) as crosslinkers, ammonium persulfate 


\section{WILEY-VCH}

(AP; Sigma, A9164) as radical initiator, and N,N,N',N'-tetramethylethylenediamine (TEMED; Sigma, T7024) as crosslinking accelerator.

The gels were prepared by dissolving $\mathrm{AAm}$ monomer powder and $\mathrm{NaCl}$ into deionized water. Molar concentrations of $\mathrm{AAm}$ and $\mathrm{NaCl}$ were fixed as $2.2 \mathrm{M}$ and $2.74 \mathrm{M}$, respectively, throughout the entire experiments. MBAA 0.06 wt.-\% and AP 0.17 wt.-\% with respect to the weight of AAm monomer were added as a cross-linker for AAm and a photo initiator, respectively. After degassing in a vacuum chamber, TEMED 0.25 wt.-\% with respect to the weight of AAm monomer were lastly added as an accelerator. The solutions were poured into a glass mold with a vacancy (100.0 x $\left.100.0 \times 0.1 \mathrm{~mm}^{3}\right)$ and covered with a $3 \mathrm{~mm}$ thick transparent glass plate. The gels were cured by the ultraviolet light cross-linker (UVC 500, Hoefer) for 20 min with $8 \mathrm{~W}$ power and $254 \mathrm{~nm}$ wavelength. The gels were then immersed in aqueous solution of the same concentration of $\mathrm{NaCl}$ for more than 24 hours. The gels absorbed more water and reached a new state of equilibrium, and the thickness of the gels was estimated to be $0.2 \mathrm{~mm}$.

The gels were cut into the desired shape by using a laser cutting system (VersaLaser VLS3.50, Universal Laser Systems) with $50 \mathrm{~W}$ power and $14 \mathrm{~cm} / \mathrm{sec}$ beam speed. Before stacking hydrogel on top of VHB, the surfaces of the hydrogels were dried with $\mathrm{N}_{2}$ gas for 1 minute to improve the adhesion between gel and VHB by removing water from the gel surfaces. A dielectric layer was sandwiched between two layers of hydrogel, resulting in a capacitive sensor. Extra two layers of VHB were attached to the top and bottom of the sensor to insulate the sensor and prevent evaporation of the hydrogel.

\section{Supporting Information}

Supporting Information is available from the Wiley Online Library or from the authors.

\section{Acknowledgements}

Work performed by JYS and ZS was funded by the NSF MRSEC award DMR-0820484. Work performed by CK (design of the study, performing of experiments, writing of the paper) was funded by the US Department of Energy, Office of Basic Energy Sciences under award DE- 


\section{WILEY-VCH}

FGo2-ooER45852. The authors thank Pierre-Marie Meyitang for assistance in experiments related to the sheet with four sensors.

Received: ((will be filled in by the editorial staff))

Revised: ((will be filled in by the editorial staff))

Published online: ((will be filled in by the editorial staff))

[1] S. Wagner, S.P. Lacour, J. Jones, P.H.I. Hsu, J.C. Sturm, T. Li, and Z.G. Suo. Physica E 2004, 25, 326.

[2] D.H. Kim, N.S. Lu, R. Ma, Y.S. Kim, R.H. Kim, S.D. Wang, J. Wu, S.M. Won, H. Tao, A. Islam, K.J. Yu, T.I. Kim, R. Chowdhury, M. Ying, L.Z. Xu, M. Li, H.J. Chung, H. Keum, M. McCormick, P. Liu, Y.W. Zhang, F.G. Omenetto, Y.G. Huang, T. Coleman, and J.A. Rogers. Science 2011, 333, 838.

[3] M.L. Hammock, A. Chortos, B.C.-K. Tee, J. B.-H. Tok, and Z.N. Bao. Adv. Mater. 2013, 25, 5997.

[4] M. Kaltenbrunner, T. Sekitani, J. Reeder, T. Yokota, K. Kuribara, T. Tokuhara, M. Drack, R. Schwodiauer, I. Graz, S. Bauer-Gogonea, S. Bauer, and T. Someya. Nature 2013, 499, 458.

[5] S. Rosset, H. R. Shea, Appl. Phys. A 2013, 110, 281-307.

[6] R. Pelrine, R. Kornbluh, Q.B. Pei, and J. Joseph. Science 2ooo, 287, 836.

[7] J.F. Zang, S. Ryu, N. Pugno, Q.M. Wang, Q. Tu, M.J. Buehler, and X.H. Zhao. Nat. Mater. 2013, 12, 321.

[8] L.B. Hu, W. Yuan, P. Brochu, G. Gruner, and Q.B. Pei. Appl. Phys. Lett. 2oo9, 94161108.

[9] D.J. Cohen, D. Mitra, K. Peterson, and M.M. Maharbiz. Nano Lett. 2012, 12, 1821.

[10] D.J. Lipomi, M. Vosgueritchian, B.C.K. Tee, S.L. Hellstrom, J.A. Lee, C.H. Fox, and Z.N. Bao. Nat. Nanotechnol. 2011, 6, 788.

[11] J.J. Liang, L. Li, X.F. Niu, Z.B. Yu, and Q.B. Pei. Nat. Photonics 2013, 7, 817.

[12] C.F. Guo, T.Y. Sun, Q.H. Liu, Z.G. Suo, and Z.F. Ren. Nat. Commun. 2014, 5, 3121. 


\section{WILEY-VCH}

[13] M.D. Dickey, R.C. Chiechi, R.J. Larsen, E.A. Weiss, D.A. Weitz, and G.M. Whitesides. Adv. Funct. Mater. 2008, 18, 1097.

[14] C. Majidi, R. Kramer, and R.J. Wood. Smart Mater. Struct. 2011, 2O, 105017.

[15] D.H. Kim, N.S. Lu, R. Ghaffari, Y.S. Kim, S.P. Lee, L.Z. Xu, J.A. Wu, R.H. Kim, J.Z. Song, Z.J. Liu, J. Viventi, B. de Graff, B. Elolampi, M. Mansour, M.J. Slepian, S. Hwang, J.D. Moss, S.M. Won, Y.G. Huang, B. Litt, and J.A. Rogers. Nat. Mater. 2011, 1o, 316.

[16] M. Ramuz, B.C.K. Tee, J.B.H. Tok, and Z.N. Bao. Adv. Mater. 2012, 24, 3223.

[17] S. Shian, R.M. Diebold, and D.R. Clarke. Opt. Express 2013, 21, 8669.

[18] C. Keplinger, J.Y. Sun, C.C. Foo, P. Rothemund, G.M. Whitesides, and Z.G. Suo. Science 2013, 341, 984 .

[19] Y.Y. Bai, Y.H. Jiang, B.H. Chen, C.C. Foo, Y.C. Zhou, F. Xiang, J.X. Zhou, H. Wang, Z.G. Suo. Appl. Phys. Lett. 2014, 104, 062902.

[20] B. Chen, J.J. Lu, C.H. Yang, J. H. Yang, J. Zhou, Y. M. Chen, and Z. Suo. ACS Appl. Mater. Interfaces 2014, 6, 7840 .

[21] J.P. Gong, Y. Katsuyama, T.Kurokawa, and Y. Osada, Adv. Mater. 2oo3, 15, 1155.

[22] J.-Y. Sun, X.H. Zhao, W.R.K. Illeperuma, O. Chaudhuri, K.H. Oh, D.J. Mooney, J.J. Vlassak, and Z.G. Suo, Nature 2012, 489, 133.

[23] A.J. Bard and L.R. Faulkner, Electrochemical methods, John Wiley \& Sons, Inc. 2001.

[24] P. Manandhar, P.D. Calvert, and J.R. Buck. IEEE Sens. J. 2012, 12, 2052.

[25] M. Kollosche, H. Stoyanov, S. Laflamme, and G. Kofod. J. Mater. Chem., 2011, 21, 8292.

[26] T.A. Gisby, B.M. O'Brien, and I.A. Anderson. Appl. Phys. Lett. 2013, 102, 193703.

[27] W.Y. Du and S.W. Yelich. Sensors \& Transducers J. 2008, 9o, 100.

[28] C. Metzger, E. Fleisch, J. Meyer, M. Dansachmuller, I. Graz, M. Kaltenbrunner, C. Keplinger, R. Schwodiauer, and S. Bauer. Appl Phys Lett 2008, 92, 013506.

[29] X.H. Zhao, W. Hong, and Z.G. Suo. Phys. Rev. B 2007, 76, 134113.

[30] A. Trols, A. Kogler, R. Baumgartner, R. Kaltseis, C. Keplinger, R. Schwodiauer, I. Graz, and 


\section{WILEY-VCH}

S. Bauer. Smart Mater. Struct. 2013, 22, 104012.

[31] E.S. Dellon, R. Mourey, and A.L. Dellon. Plast. Reconstr. Surg. 1992, 9o, 112. 
a Voltage-off state

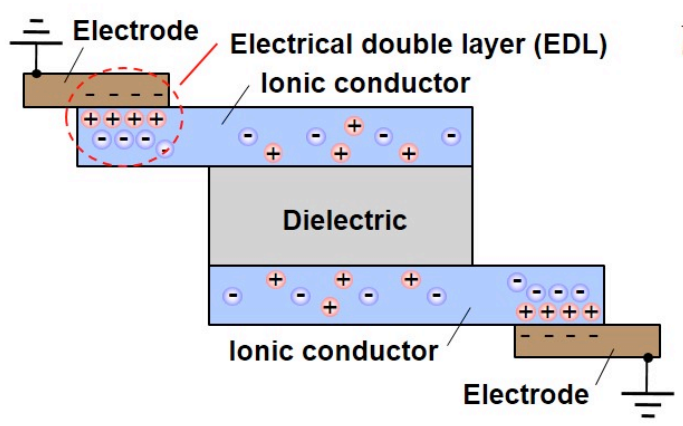

d

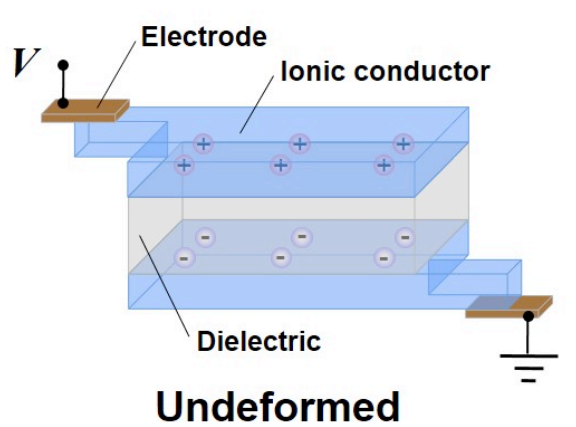

b Voltage-on state

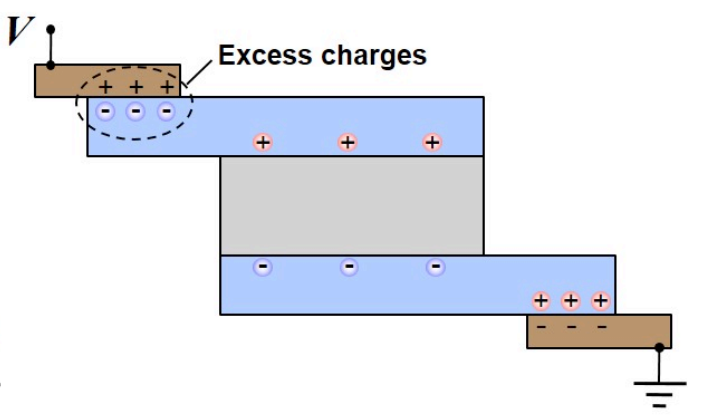

e

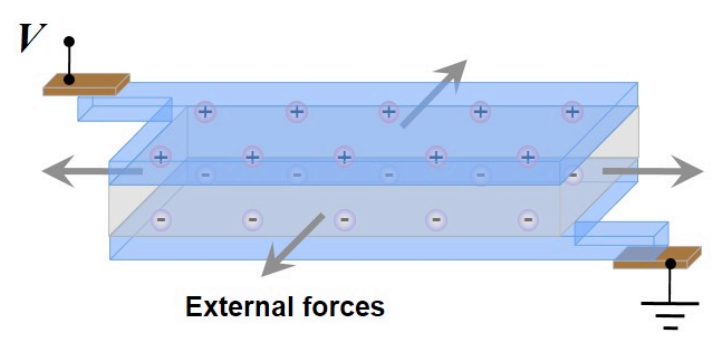

Deformed

Figure 1. Basic design of ionic skin. (a) A stretchable dielectric is sandwiched between two stretchable ionic conductors. Outside the deformable area, the ionic conductors connect to metallic electrodes. Even when both electrodes are grounded, electrical double layers form at the interfaces between the electrodes and the ionic conductors, as well as at the interfaces between the ionic conductors and the dielectric. (b) When a voltage is applied between the two electrodes, the interfaces accumulate additional electric charges. Only the excess charges are shown in the figure. (c) Equivalent electrical circuit. (d) In the absence of external forces, a small voltage is applied to measure the capacitance. (e) When external forces stretch the structure, the capacitance increases. 


\section{WILEY-VCH}

a

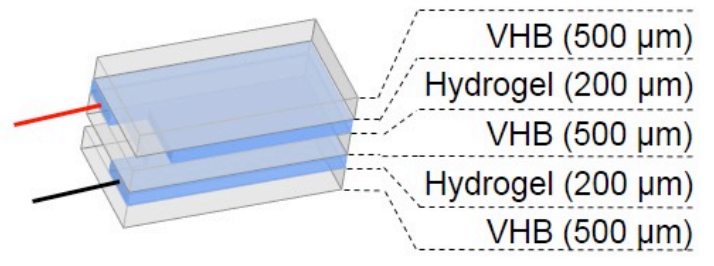

d

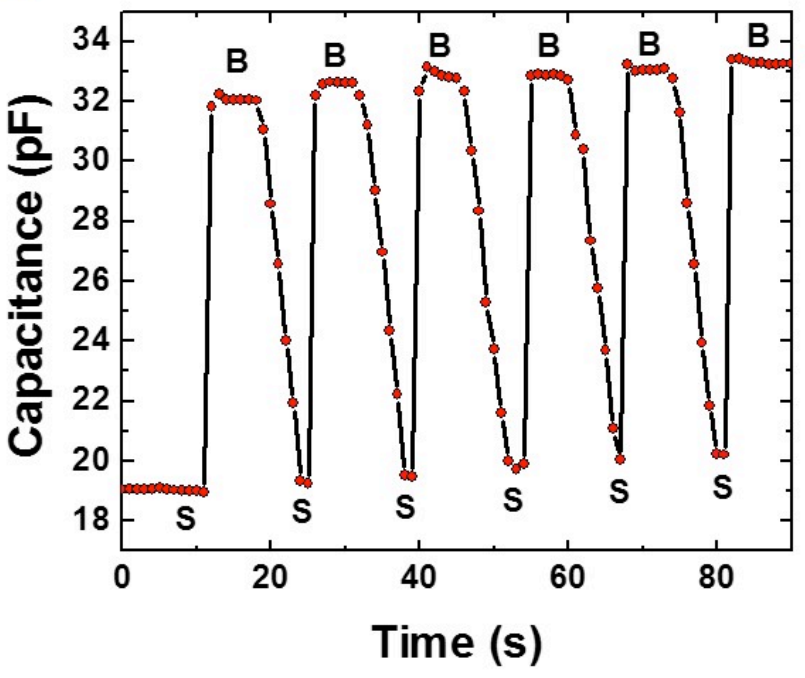

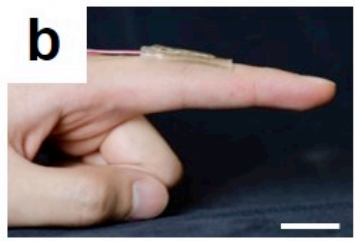

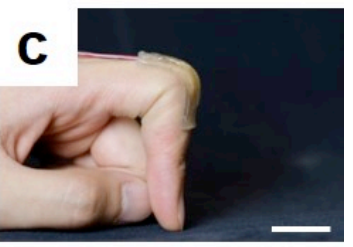

e

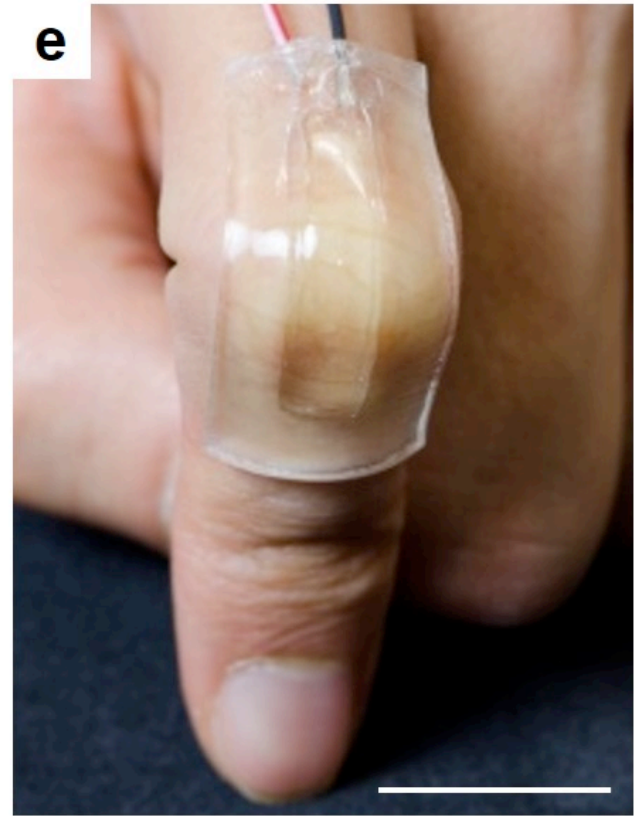

Figure 2. Ionic skin used as a strain sensor. (a) A strain senor was fabricated by sandwiching a layer of stretchable dielectric (VHB 4905 tape, 3M) between two layers of a stretchable ionic conductor (salt-containing hydrogel), which were then connected to two metallic electrodes. The device was covered with two additional layers of VHB. (b) The strain sensor was attached to a straight finger. (c) The bending of the finger stretched the strain senor. (d) The capacitance was measured as the finger bent cyclically. 'B' denotes bent, and ' $\mathrm{S}$ ' denotes straight. (e) The strain senor was fully transparent. The scale bars in (b), (c) and (e) are $2 \mathrm{~cm}$. 

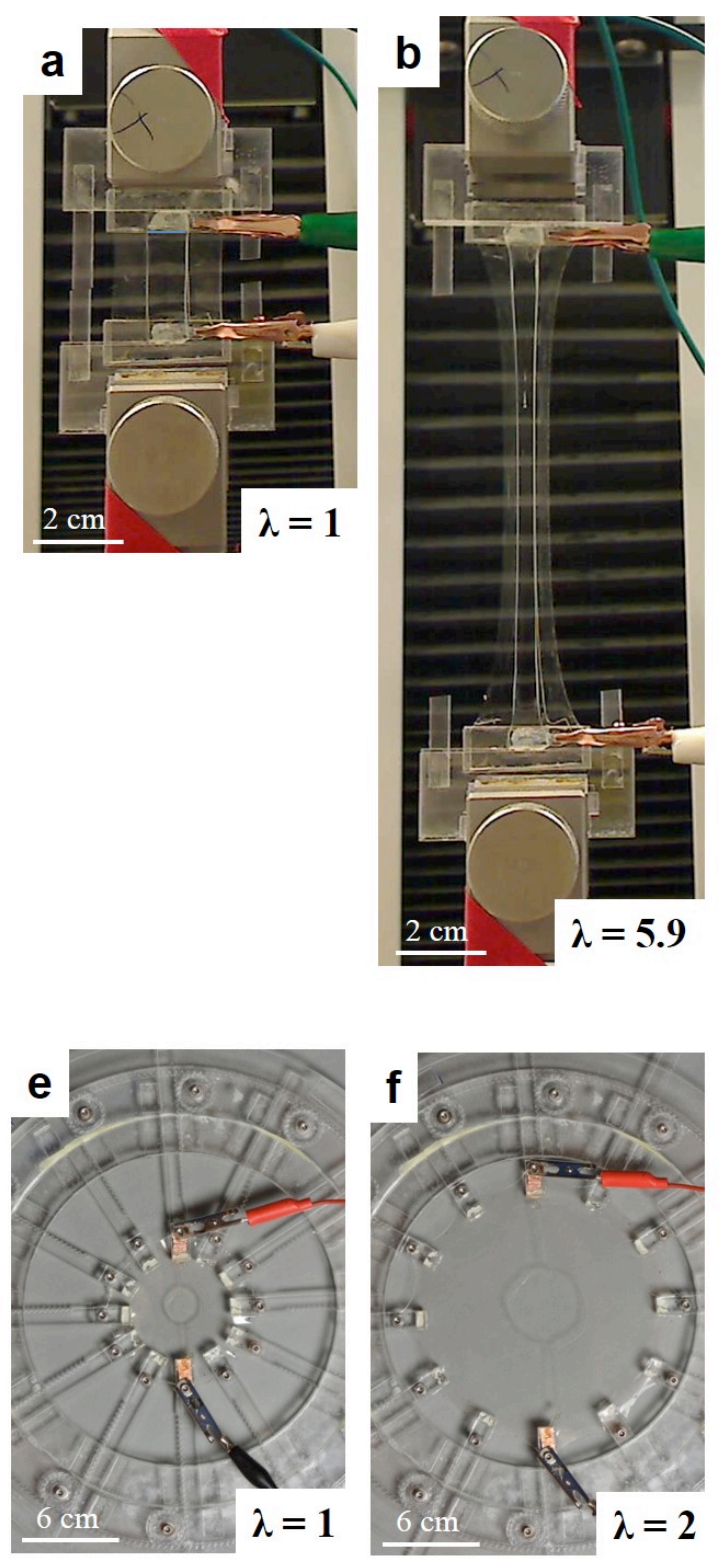

C

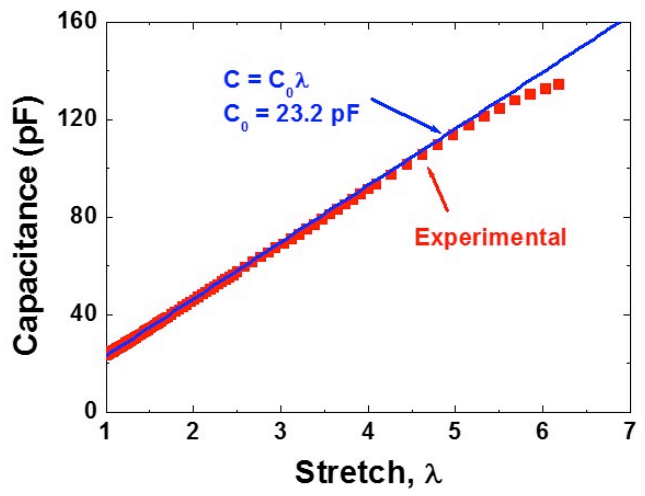

d

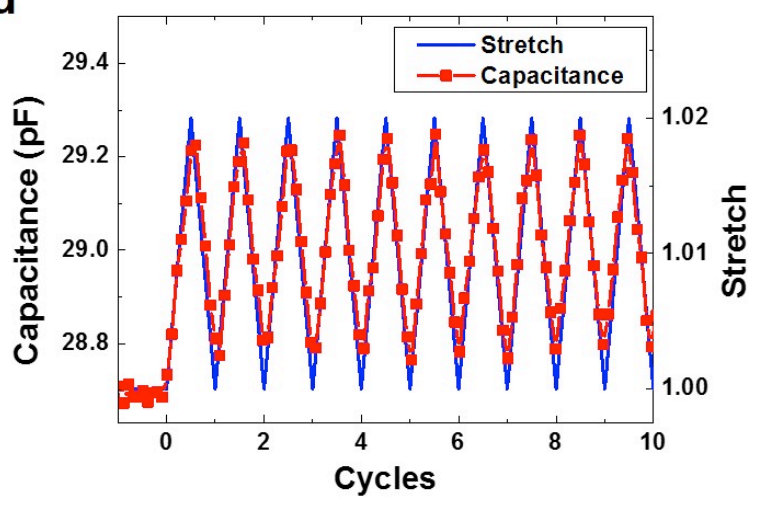

g

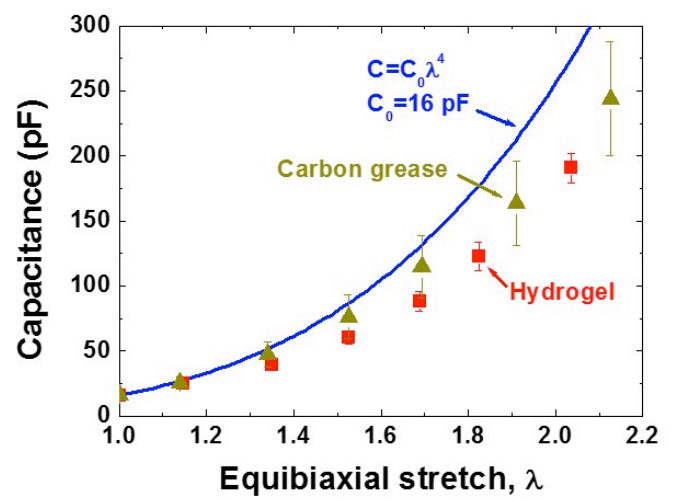

Figure 3. Strain sensors under uniaxial and equal-biaxial forces. A uniaxial force stretched a strain sensor from an undeformed state (a) to a deformed state (b). The stretch, $\lambda$, is defined by the distance between the two clamps when the sensor is deformed, divided by the distance when the sensor is undeformed. (c) Experimental data for capacitance and stretch were compared with a theoretical prediction. (d) A uniaxial force loaded the strain sensor cyclically at a frequency of $1 \mathrm{~Hz}$ between the undeformed state and a stretch of 1.02. A homemade device pulled the strain sensor from an undeformed state (e) to a state of equal-biaxial stretch (f). (g) Experimental data of strain sensors under equal-biaxial forces were compared with a theoretical prediction. The figure also compares strain sensors using hydrogel as conductors to strain sensors using carbon grease as conductors. 
a

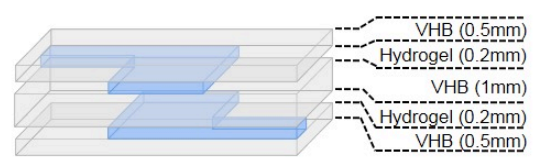

b
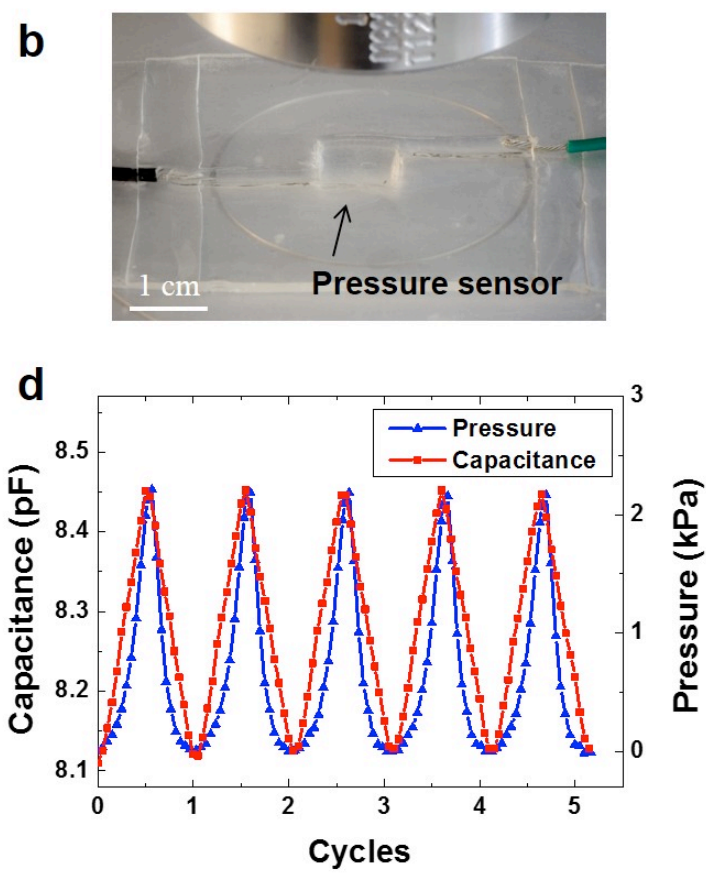

C
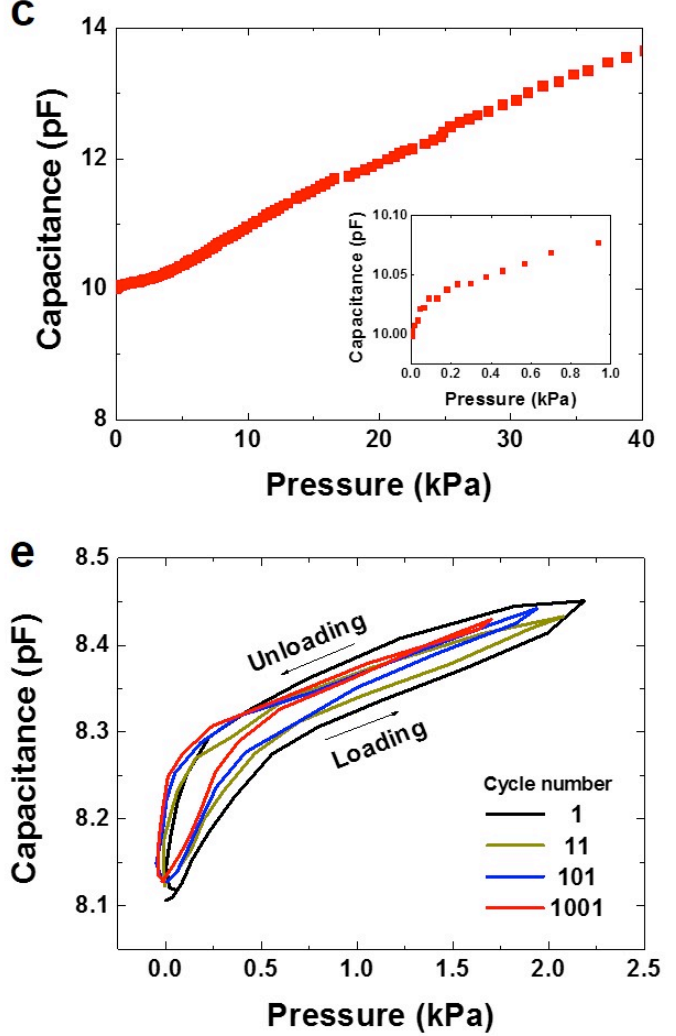

Figure 4. Ionic skin used as a pressure sensor. (a) Design of the pressure sensor. (b) The pressure sensor was placed on a stage of a mechanical testing machine. (c) The measured capacitance-pressure curve. The inset shows that the pressure sensor had high resolution in the range of $1 \mathrm{kPa}$, sensitive enough to detect a gentle touch of a finger. (d) The pressure and capacitance of a sensor cyclically compressed to a strain of $1 \%$, at a frequency of $0.5 \mathrm{~Hz}$. (e) Pressure-capacitance curves were measured for more than 1000 cycles. Four representative cycles are shown. 


\section{WILEY-VCH}
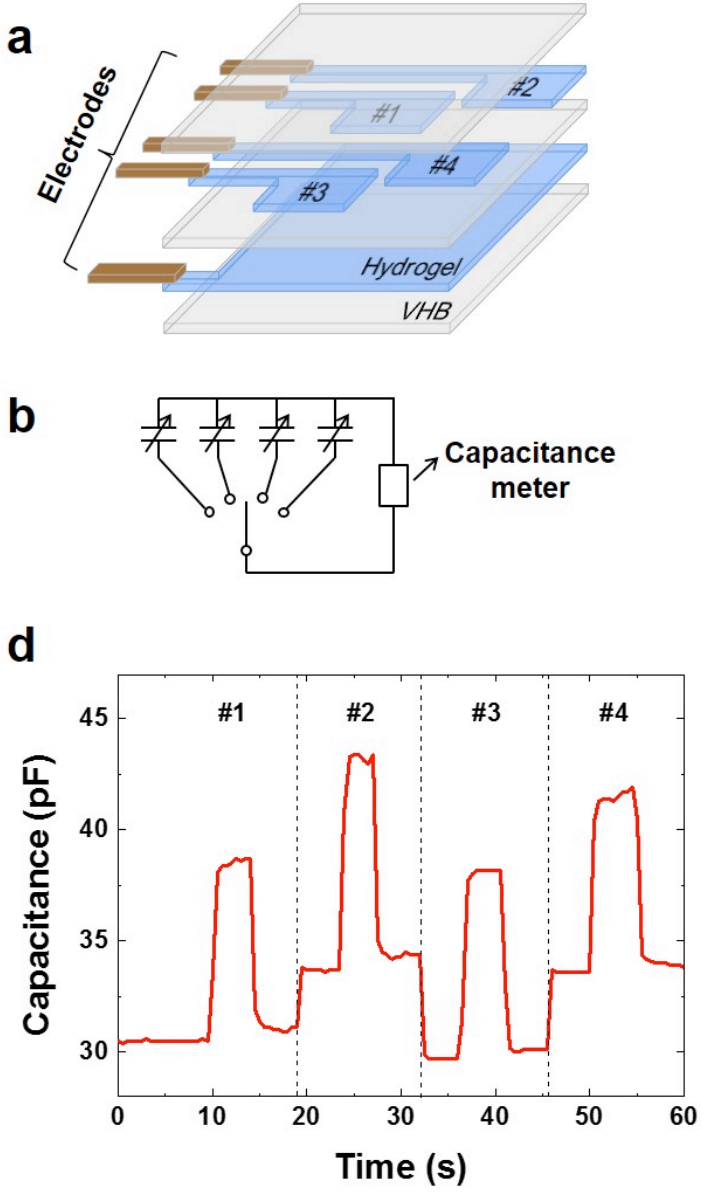

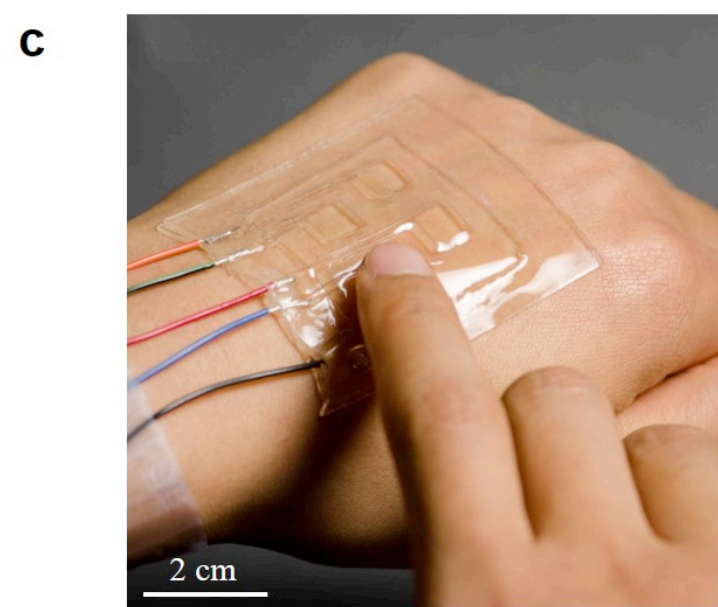

e

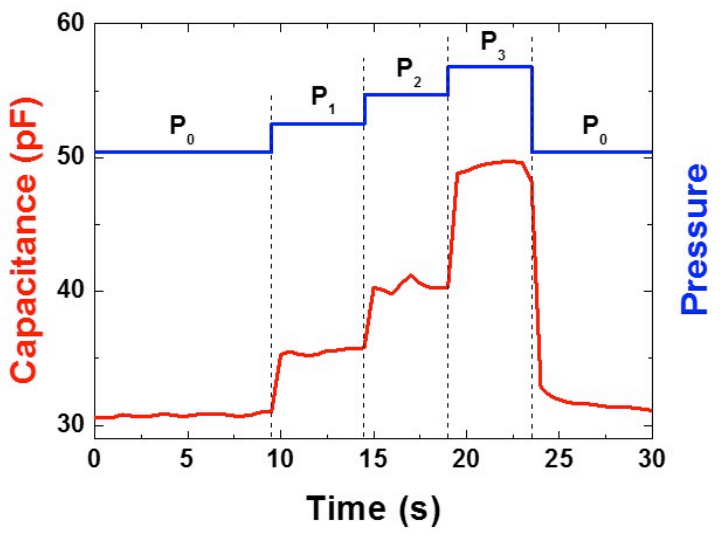

Figure 5. An array of sensors reports the location and pressure of touch. (a) An ionic skin contained four pressure sensors, made of four small areas of hydrogel on top of a dielectric, and a large area of hydrogel beneath the dielectric. The large area of hydrogel served as the common ground of the four sensors. Each small area of hydrogel was connected to the external electronic circuit through a thin line of hydrogel. Two additional layers of dielectric covered the ionic skin. (b) The four sensors were connected through a rotational switch to a capacitance meter. (c) The sensor array was attached on the back of a hand, and one sensor was pressed with a finger. (d) The ionic skin detected the location of touch. When the capacitance meter connected to a specific sensor, say sensor \#1, the capacitance took a baseline value before a finger pressed the sensor, increased to a high value when the finger pressed, and then returned to the baseline value when the finger no longer pressed. The baseline values for sensors \#1 and \#3 were lower than those of \#2 and \#4 because the latter had longer connecting lines of hydrogel overlapping with the ground hydrogel. (e) The ionic skin detected the pressure of touch. When a finger pressed a single sensor and changed the pressure from $P_{0}=0$ to $P_{1}, P_{2}, P_{3}$, and back to $P_{0}$, the capacitance also changed. 


\section{WILEY-VCH}

Electronic skins (i.e., stretchable sheets of distributed sensors) report signals using electrons, whereas natural skins report signals using ions. Here ionic conductors are used to create a new type of sensory sheet, called "ionic skin". Ionic skins are highly stretchable, transparent, and biocompatible. They readily measure strains from $1 \%$ to $500 \%$, and pressure as low as $1 \mathrm{kPa}$.

\section{Keywords}

Strain sensors, pressure sensors, stretchable electronics, ionic conductors, electronic skin

Jeong-Yun Sun, Christoph Keplinger, George M. Whitesides*, and Zhigang Suo*

\section{Ionic skin}

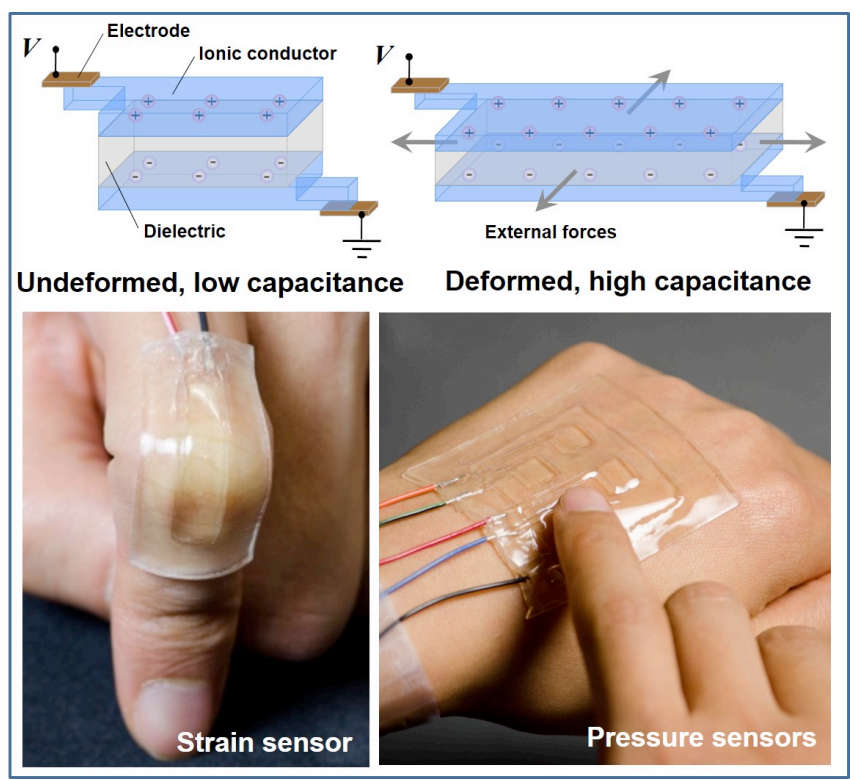




\section{WILEY-VCH}

Copyright WILEY-VCH Verlag GmbH \& Co. KGaA, 69469 Weinheim, Germany, 2013.

Supporting Information

Ionic skin

Jeong-Yun Sun, Christoph Keplinger, George M. Whitesides*, and Zhigang Suo*

\section{Supplementary Video 1. A sensor detects the bending of a finger.}

We attached a sensor to a finger, and used two metallic wires to connect the sensor to a capacitance meter. When the finger bent, the capacitance meter indicated the increase of the capacitance of the sensor, and the watch indicated the time. As the finger bent repeatedly, the sensor remained transparent, and conformed to the movement of the joint.

\section{Supplementary Video 2. An equibiaxial stretcher.}

We designed and fabricated a device to pull a thin sheet equibiaxially.

\section{Supplementary Video 3. An ionic skin detects the location of touch.}

We attached an ionic skin containing four sensors to the back of a hand, and used a rotational switch to connect a capacitance meter to the sensors one at a time. The capacitance was recorded through a Labview (National Instruments) program, and the capacitance of the connected sensor was displayed on the screen of a laptop as a function of time. When the capacitance meter connected to a specific sensor, the capacitance took a baseline value before a finger pressed the sensor, increased to a high value when the finger pressed, and returned to the baseline value after the finger was removed from the sensor. The baseline values of the four sensors were different because the sensors had hydrogel interconnects of different lengths.

\section{Supplementary Video 4. An ionic skin detects the pressure of touch.}


We connected the capacitance meter to a specific sensor. When a finger applied several levels of pressure on the sensor, the capacitance of the sensor changed.

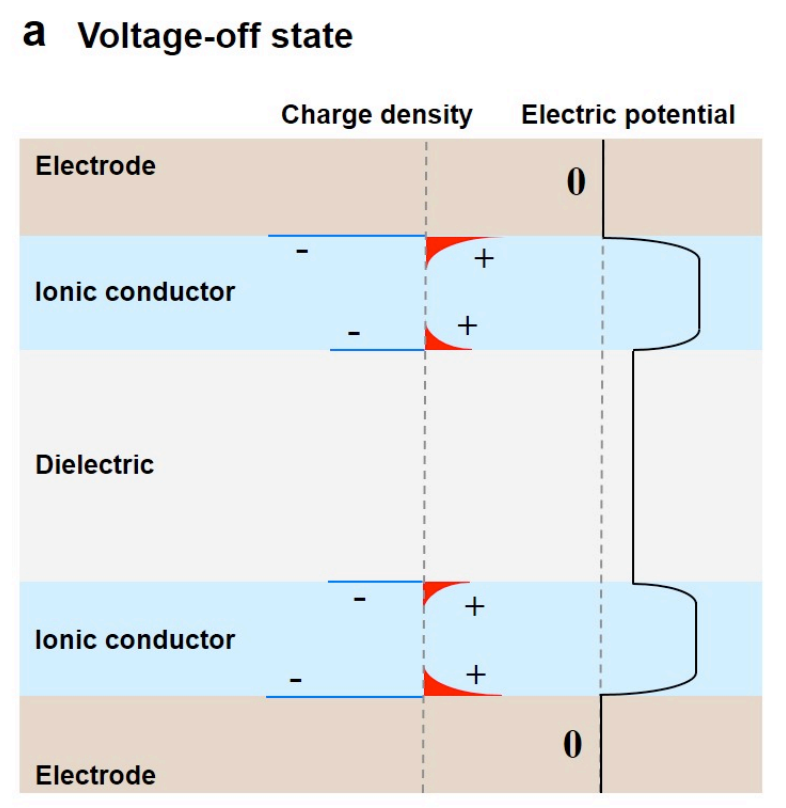

\section{b Voltage-on state}

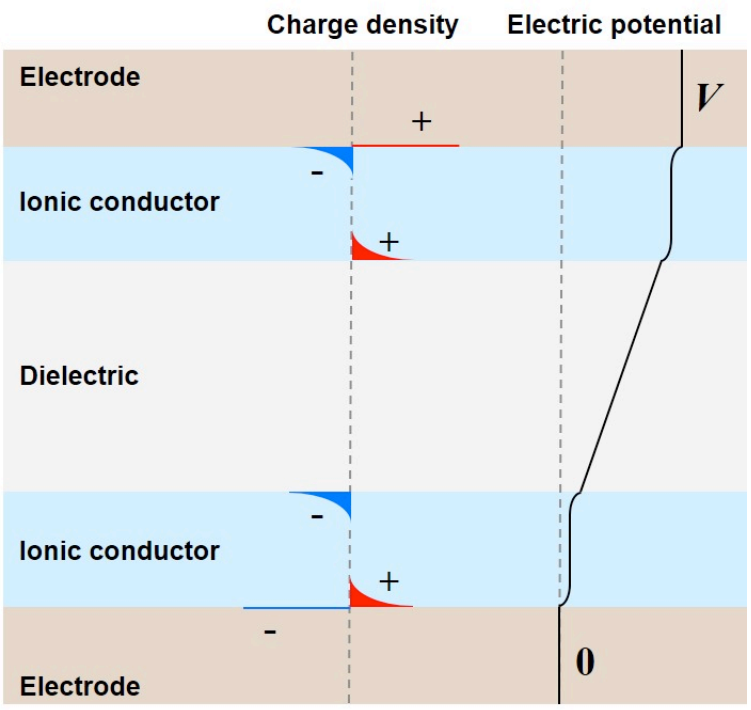

Figure S1 | Distributions of electric charge and potential across the structure. (a) In the voltage-off state, the two electrodes are grounded, and the distributions of charge density and electric potential are symmetric with respect to the centerline of the dielectric. Trapped electrons and adsorbed ions may localize within molecular distance from the interface between a metallic electrode and an ionic conductor. This localized layer has a net electric charge; the net amount and the polarity of the localized charge depend on the two materials. The drawing assumes the net localized charge to be negative, indicated by a blue line. This localized net charge is shielded by mobile ions in the ionic conductors. Because the concentration of the mobile ions in the ionic conductor is typically much lower than the concentration of free electrons in the metallic electrode, the shielding charge in the ionic conductor is diffused, decaying over the distance of the Debye length. Similarly, a layer of net charge may also localize with a molecular distance from the interface between a dielectric and an ionic conductor. The 


\section{WILEY-VCH}

localized charge is shielded by diffused charge in the ionic conductor. The drawing assumes that the dielectric is perfect and has no mobile charges. The thicknesses are not drawn to scale. The charge density and electric potential near the interfaces vary within the Debye length (some nanometers), but the thicknesses of the dielectric and ionic conductor are of hundreds of microns. (b) In the voltage-on state, the two electrodes are subject to a voltage $V$. The drawing only shows the excess electric charges and electric potential caused by the applied voltage. The distributions are anti-symmetric with respect to the centerline of the dielectric. The two interfaces between the electrodes and the ionic conductors behave as capacitors, in series with the capacitor made of the dielectric. The charges on the three capacitors are equal.

a

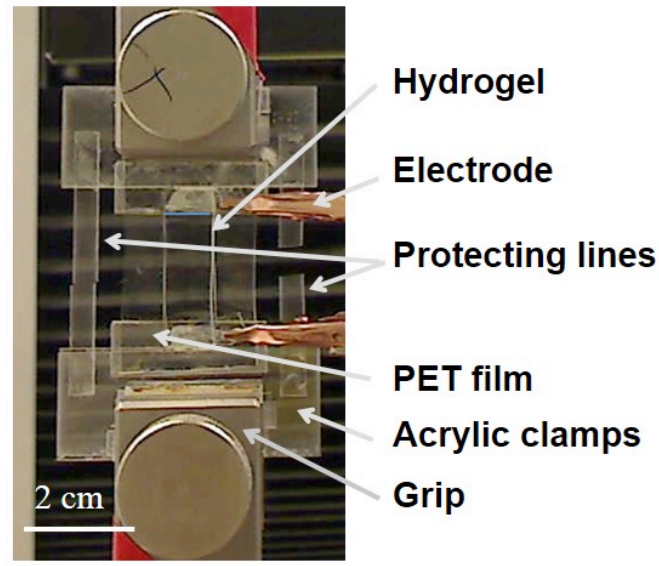

b

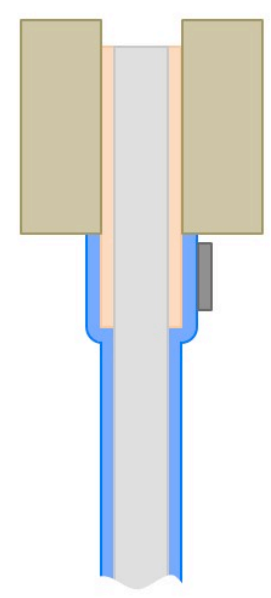

Hydrogel Dielectric (VHB) PET film Clamps (acrylic) Electrode

Figure S2 | Design of a strain sensor for uniaxial tensile tests. A strain sensor was fabricated with a special design for a uniaxial tensile test. (a) The sensor was soft, and was fixed to stiff acrylic clamps using a glue (Instant Krazy Glue, Krazy Glue). Furthermore, two stiff protecting lines were glued at the edge of acrylic clamps to prevent a deformation before loading. The protecting lines were cut after placing the sample between the grips. (b) The cross section of the grip. To avoid sliding between the hydrogel, we used the glue to bond a $100 \mu \mathrm{m}$ thick PET film to the acrylic and the hydrogel. 

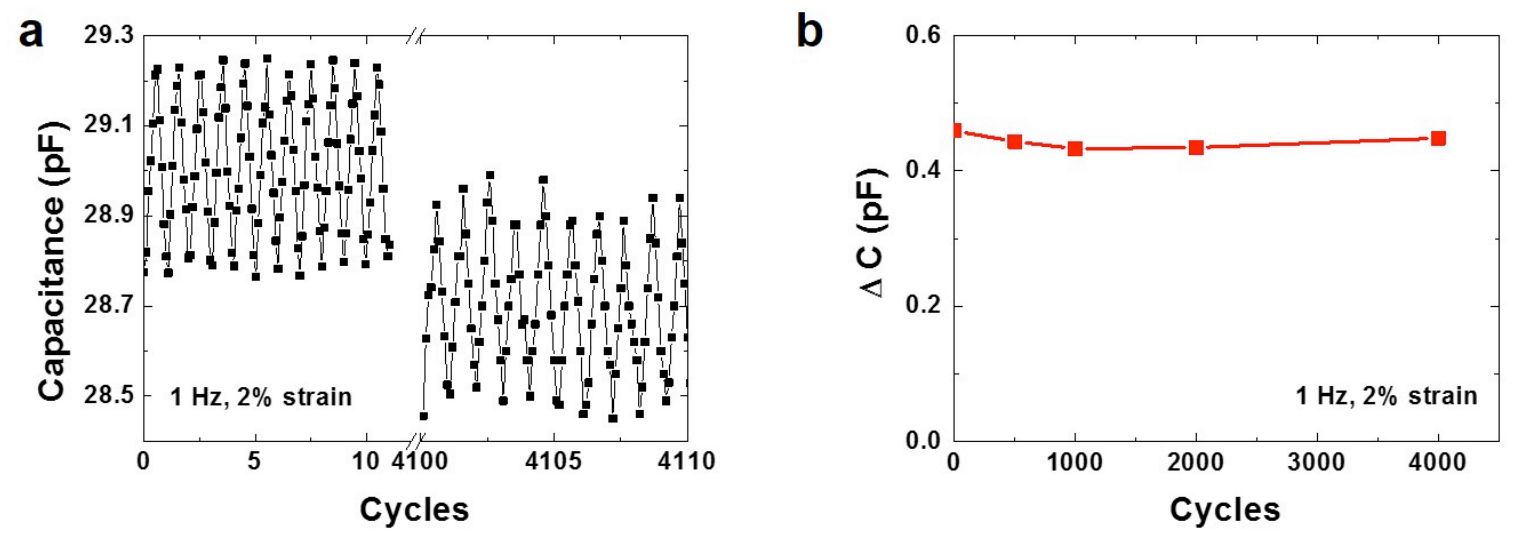

Figure S3 | Characteristics of strain sensors under uniaxial cyclic loading. A strain sensor was subject to uniaxial cyclic loading at a frequency of $1 \mathrm{~Hz}$ and a maximum strain of $2 \%$. (a) The capacitance $C$ of the sensor was recorded using a capacitance meter. The capacitance drifted slightly over cycles. For example, the minimum value of the capacitance of each cycle, $C_{\min }$, was $28.77 \mathrm{pF}$ in the initial undeformed state, and was $28.46 \mathrm{pF}$ at cycle number 4110 . (b) The change in capacitance in each cycle, $\Delta C=C_{\max }-C_{\min }$, varied less than $5 \%$ over 4000 cycles. 


\section{WILEY-VCH}
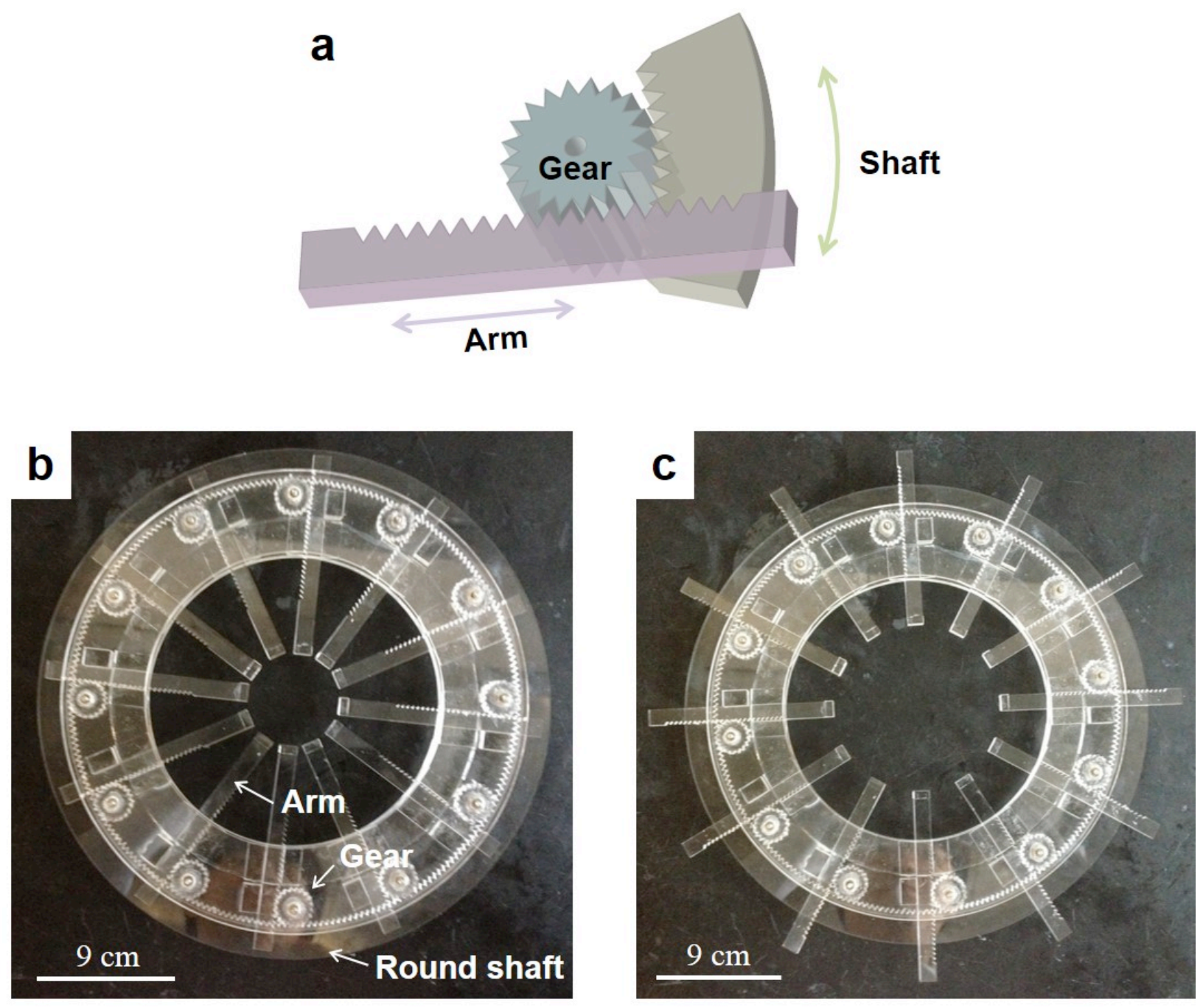

Figure S4 | Equibiaxial stretcher. An equibiaxial stretcher was made of a $3 \mathrm{~mm}$ thick acrylic plate (McMaster-Carr, 8560k239). The acrylic plate was cut into the designed shape by using a laser cutting system (VersaLaser VLS3.50, Universal Laser Systems) with 50 W power and $0.84 \mathrm{~cm} / \mathrm{sec}$ beam speed. When the round shaft is rotated, the rotation is transferred to an arm through a gear. Since the rotation of the shaft is transferring the same amount of movement to 12 arms, the rotation will cause equibiaxial stretching. The stretch can be readily controlled up to 3 . 


\section{WILEY-VCH}

a

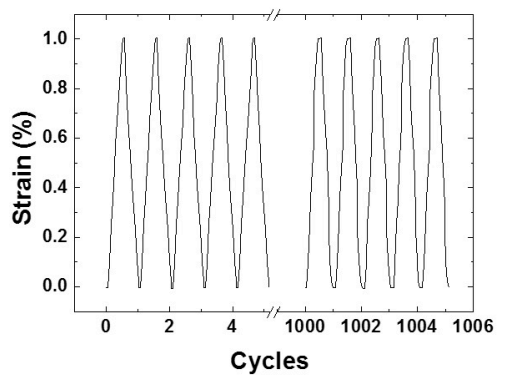

b

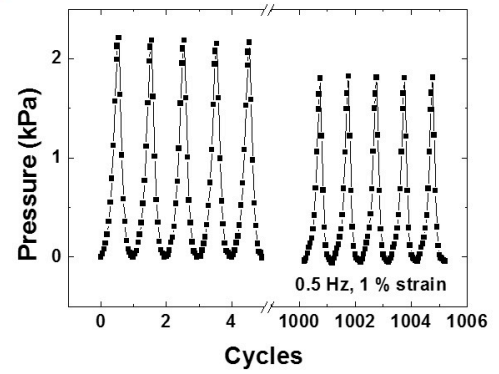

C

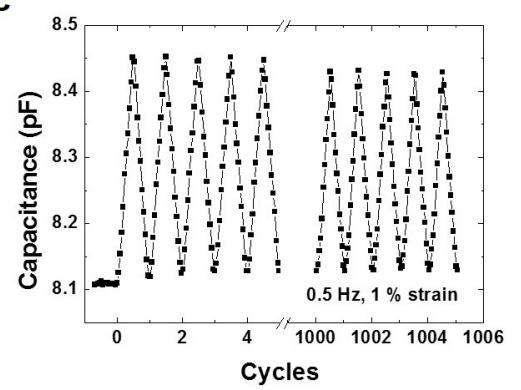

Figure S5 | Characteristics of a pressure sensor under cyclic loading. (a) A pressure sensor was cyclically compressed with a prescribed strain of $1 \%$ and a frequency of $0.5 \mathrm{~Hz}$ over 1000 cycles. (b) The pressure was measured by a load cell. The pressure decreased somewhat over the cycles, possibly due the viscoelasticity of the materials. (c) The capacitance $C$ of the sensor was monitored by a capacitance meter. In each cycle, let $\Delta C$ be the difference in capacitance before and after loading. The difference in capacitance $\Delta C$ drifted from $0.34 \mathrm{pF}$ to $0.3 \mathrm{pF}$ over 1000 cycles. 